\title{
The impact of extent of resection in surgical outcome of pilomyxoid astrocytoma: a case study
}

\author{
Dipak Chaulagain ${ }^{1,2}$, Volodymyr I. Smolanka ${ }^{1,2}$, Andriy V. Smolanka ${ }^{1,2}$, Taras S. Havryliv ${ }^{1,2}$
}

${ }_{1}$ Uzhhorod Regional Clinical Center of Neurosurgery and Neurology, Uzhhorod, Ukraine

2 Neurosurgery Department, Uzhhorod National University, Uzhhorod, Ukraine

Received: 20 October 2021 Accepted: 19 November 2021

Address for correspondence: Dipak Chaulagain, Neurosurgery Department, Uzhhorod National University, 24 Kapushanska Str. Uzhgorod, 88018, Ukraine, e-mail: neurodipak@gmail.com
The pilomyxoid astrocytoma (PMA) is a rare glioma that has recently been identified as a separate entity and is frequently found in the hypothalamic region. PMA is a subtype of pilocytic astrocytoma (PA), with clinical, histological, and molecular data indicating a close relationship as well as more aggressive biological behaviour in the former. There is still doubt in surgical outcome of PMA that the extent of resection, independent of location or age, is a key factor of recurrence and subsequent therapeutic choices. However, further study is needed to better understand its behaviour and, as a result, establish a consensus on its management.

This research features a 2-year-6-month-old female who sought medical attention after complaining of weight loss for four weeks and vomiting for two weeks prior to her visit to the doctor. She had no additional symptoms. Only bilateral pailledema was found during the physical examination. The magnetic resonance imaging (MRI) scans revealed a tumor in the sellar area with heterogeneous enhancement. The patient had ventriculoperitoneal (VP) shunting followed by partial tumor excision twice (Extent of resection 35 percent followed by 16 percent as total 51 percent). The histology and immunohistochemical investigations revealed typical PMA characteristics. Adjuvant treatment, which included chemotherapy and radiosurgery, was initiated for the patient. She has been asymptomatic for two years and has showed no indications of progression of the disease on follow-up scans.

Keywords: astrocytoma; extent of resection; pilomyxoid astrocytoma; glioma; pilocytic astrocytoma

\section{Introduction}

Pilomyxoid astrocytoma (PMA) is a highly rare tumor that deserves to be recognized as a unique and distinct entity. The characteristics of PMA are similar to those of pilocytic astrocytoma (PA), the most frequent central nervous system (CNS) tumor in children. However, significant pathological abnormalities have been identified that distinguish these two entities. Previous research has revealed that PMA behaves more aggressively than PA, with shorter overall survival and a greater rate of recurrence and spread [1]. It usually occurs in infant and young children and is located in the hypothalamic/chiasmatic area. Pilomyxoid tumors can occasionally cause hemorrhage in these locations [2]. In contrast to the normal biphasic histology of pilocytic astrocytomas, these neoplasms have monomorphous piloid cells in a loose fibrillary and myxoid background [3]. Pilomyxoid astrocytomas are solid enhancing lesions that may be differentiated from pilocytic astrocytomas by considerably greater $\mathrm{rCBV}$, as well as dynamic susceptibility contrast perfusion and diffusion weighted imaging, and have a higher rate of leptomeningeal involvement and dissemination [4].

The treatment of children low-grade astrocytomas, including PMA, is still debatable. Surgery is frequently the primary therapeutic option, depending on the location of the lesion. Cerebellar tumors, for example, are frequently treated by full resection, but hypothalamic and suprasellar tumors are not typically susceptible to gross total resection (GTR). GTR is the most consistent predictor of positive prognosis in the pediatric population in low-grade gliomas, assuming it can be done without undue morbidity. As a result, the long-term biological activity of remaining neoplastic tissue influences the utilization of adjuvant treatment to treat these lesions. PMA is no exception when it comes to the indications for adjuvant therapy in the treatment of low-grade astrocytomas. Adjuvant treatment, either chemotherapy or radiation, is commonly used in at least three circumstances: 1) tumor recurrence after first GTR; 2) partly resected tumors producing neurological damage; and 3) partially resected tumors exhibiting growth on follow-up imaging even in the absence of symptoms [1]. We present a case of PMA affecting the sellar area in a 2-year-6-month-old female who presented with increased intracranial pressure (ICP) characteristics and bilateral papilledema.

\section{Case Report}

A 2 year-6-month old female patient, previously losing weight, presented to Uzhhorod Regional Neurology and Neurosurgical Center for treatment complaining 
vomiting for a period of over 2 weeks. Patient had a history of losing weight which started 1 year before her current presentation and were progressively worsening. Parents denied having focal neurological deficits or any other symptoms. The neurological examination revealed only bilateral papilledema. Clinical examination revealed that the patient had no focal neurological deficit and her Karnofsky Performance Status (KPS) was 100. Furthermore, the patient had early a bilateral papillary

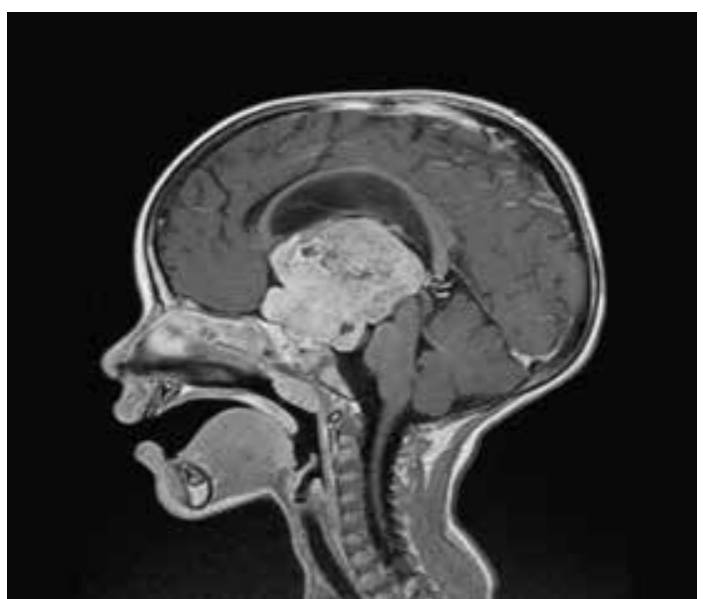

Fig. 1. T1-weighted MRI of head showed sellar region tumor with heterogeonous enhancement with mass effect and hydrocephalus)
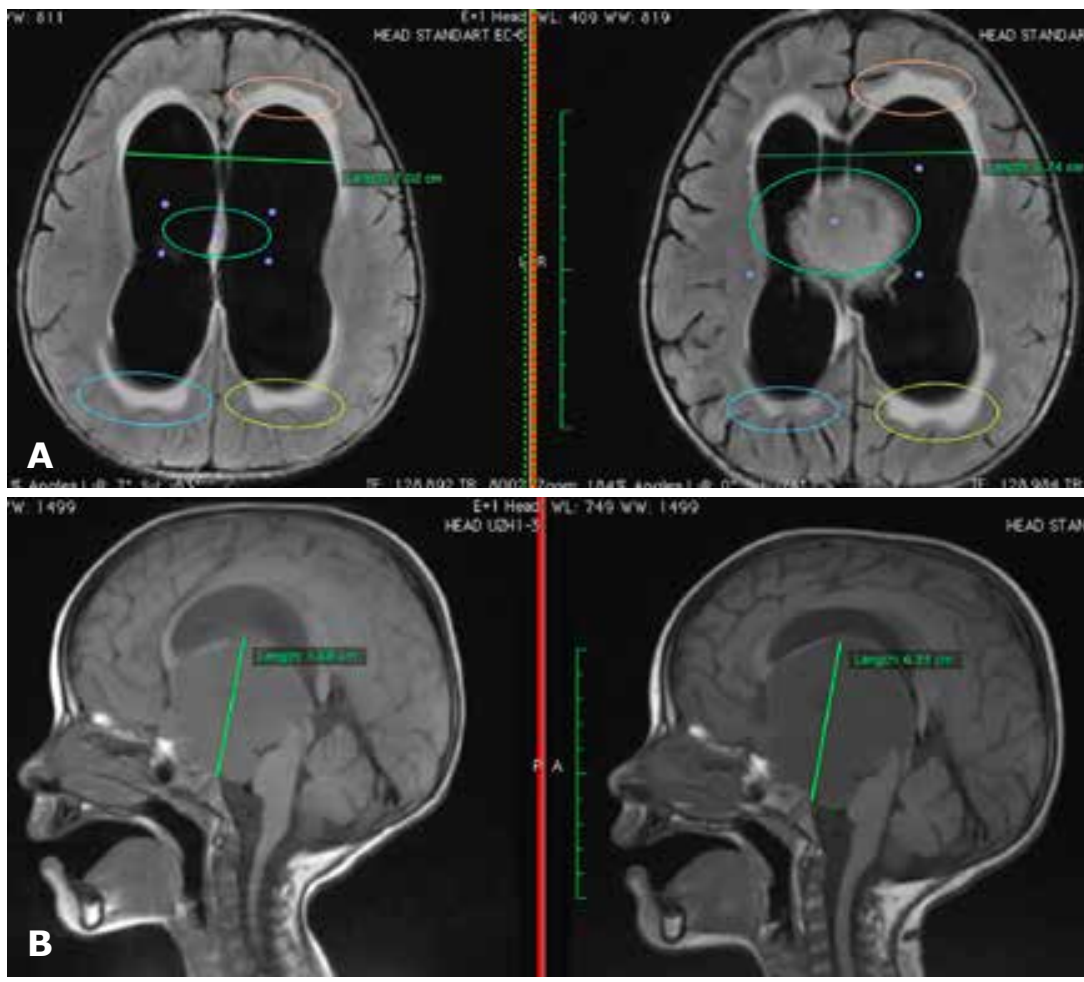

Fig. 2. A - MRI of brain axial (fluid attenuated inversion recovery-FLAIR) showed pre and post-vp shunt); B - T1-weighted MRI (sagittal) of brain showed pre and post-vp shunt)
1000 Sta

edema which was found on fundoscopy. All laboratory data upon admission were normal.

Magnetic resonance imaging (MRI) of the head displayed sellar region tumor with heterogenous enhancement. There was also associated with mass effect and hydrocephalus (Fig. 1).

The patient underwent ventriculoperitoneal (VP) shunt to treat hydrocephalus and intracranial hypertension (Fig. 2A, 2B). After VP shunt, there was improvement of her symptoms.

After 2 weeks, she underwent another surgical procedure through frontal craniotomy with transcallosal approach, which resulted in partial removal of tumor and extent of resection was calculated by volumetric measurement method. Area was calculated from FLAIR MRI of brain where pre-operation area of tumor (a) was $133.791 \mathrm{~cm} 2(3.841+3.841+24.522+20.772+16.359+22$ $.547+21.490+12.168+6.151+2.100)$ and post-operation area of tumor (b) was $93.049 \mathrm{~cm} 2(2.858+2.858+9.99$ $2+7.271+7.578+21.695+20.233+12.601+6.055+1.908)$. Then, Amount of tumor removed (c) was calculated by subtracting from $a$ to $b$ equals to $40.742 \mathrm{~cm} 2$. For the extent of resection was calculated as $c * 100 / a$ equals to $30.45 \%$ (Fig. 3).

After 6 months, she underwent second time surgical procedure through the same approach, which resulted in partial removal of tumor and extent of resection was $16.17 \% \%$, where also the same volumetric method was implicated to calculate for the extent of resection. Area was calculated from T1-contrast MRI of brain where pre-operation area of tumor (a) was $139.306 \mathrm{~cm} 2(2.808+2.808+$ $10.246+13.899+19.374+21.728+2$ $3.060+17.966+19.256+8.161)$ and post-operation area of tumor (b) was $116.77 \mathrm{~cm} 2(2.891+2.891+9$. $265+12.988+16.391+20.418+18.3$ $07+13.096+11.951+8.572)$. Then amount of tumor removed (c) was calculated by subtracting from a to $b$ equals to $22.536 \mathrm{~cm} 2$. For the extent of resection was calculated as c*100/a equals to $16.17 \%$ (Fig.4). So, all together $46.62 \%$ extent of resection was done as a partial resection. After 2 months, due to the valve extrusion, VP shunt was removed from the patient.

The histological examination identified an astrocytic tumor with extensive fibrillary process, Rosenthal fibers and protein droplets. Tumor cells laid often in a mucoid matrix. Tumor cell architectures also included perivascular growth and pseudorosettes. The immunohistochemical analysis was positive for GFAP and MAP2

This article contains some figures that are displayed in color online but in black and white in the print edition 


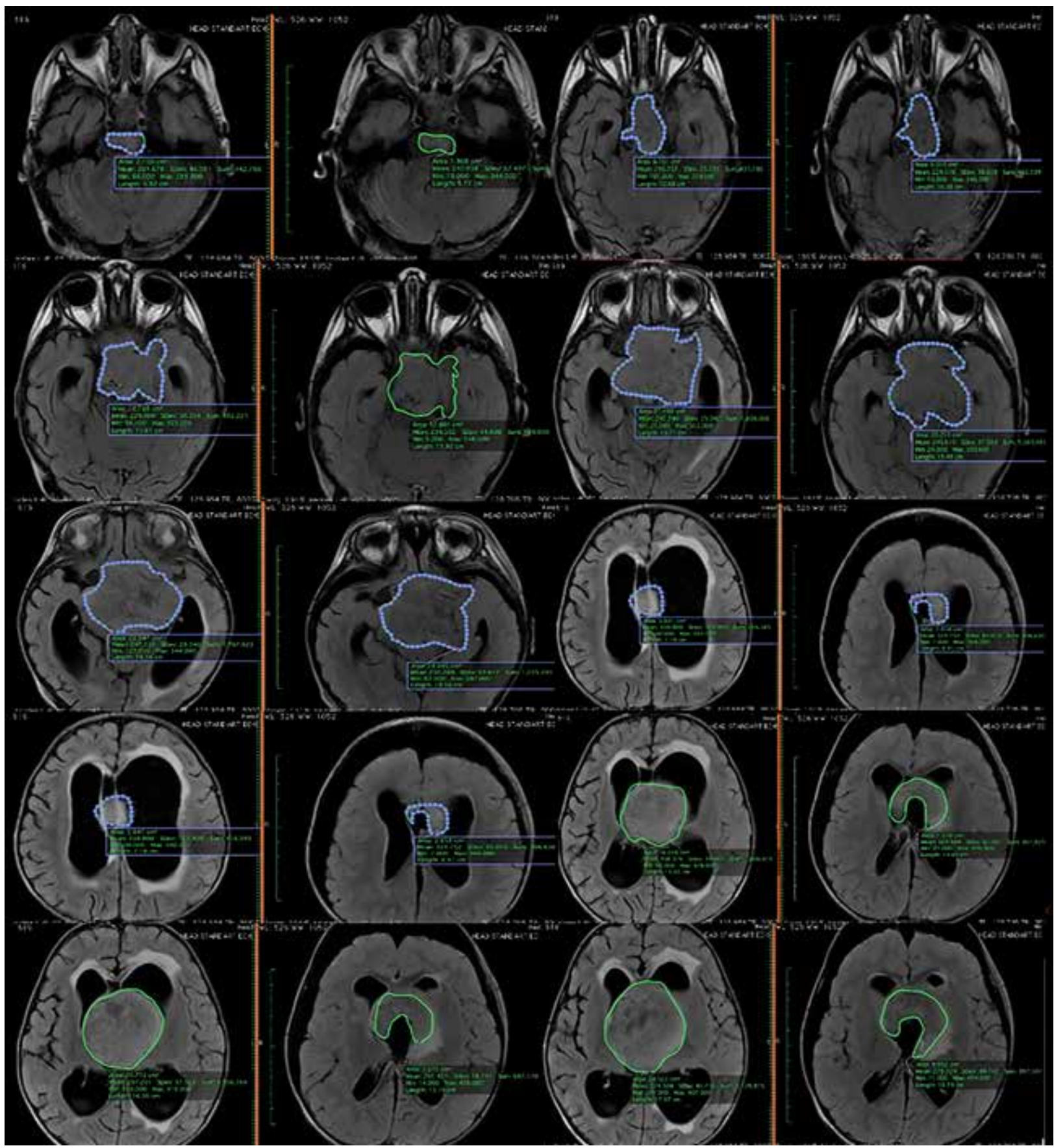

Fig. 3. FLAIR MRI of brain (axial) of pre and post-first operation with volumetric calculation after partial removal of tumor)

epitopes. There was no staining of BRAF and IDH1 mutation specific antibodies and proliferation activity was below the $5 \%$ margin. Histopathology and immunohistochemical study show the characteristic appearance of a pilocytic matrix with mucoid areas and perivascular growth.

After 2nd surgery, the patient started chemotherapy with dabrafenib as adjuvant therapy associated with radiosurgery. The patient has been in follow-up until the present date and she has been asymptomatic so far and her KPS was 100. The follow-up MRI scan after 3 years has shown no sign of progressions of the remaining lesion (Fig.5).

\section{Discussion}

The PMA is a rare central nervous system tumor that was originally identified in 1999. Previously, the PMA was categorized as a pilocytic astrocytoma due to some of its features (PA). Recent research, however, has distinguished PMA and PA as two separate entities. Nonetheless, other writers regard PMA to be a variation of PA, considering PMA as part of the normal maturation process of PA [5].The WHO classified it as a grade II tumor in 2007. However, according to the 2016 modification of the WHO classification, PMA should not be automatically classified to this tumoral grade. PMA can be seen everywhere in the central nervous system, 


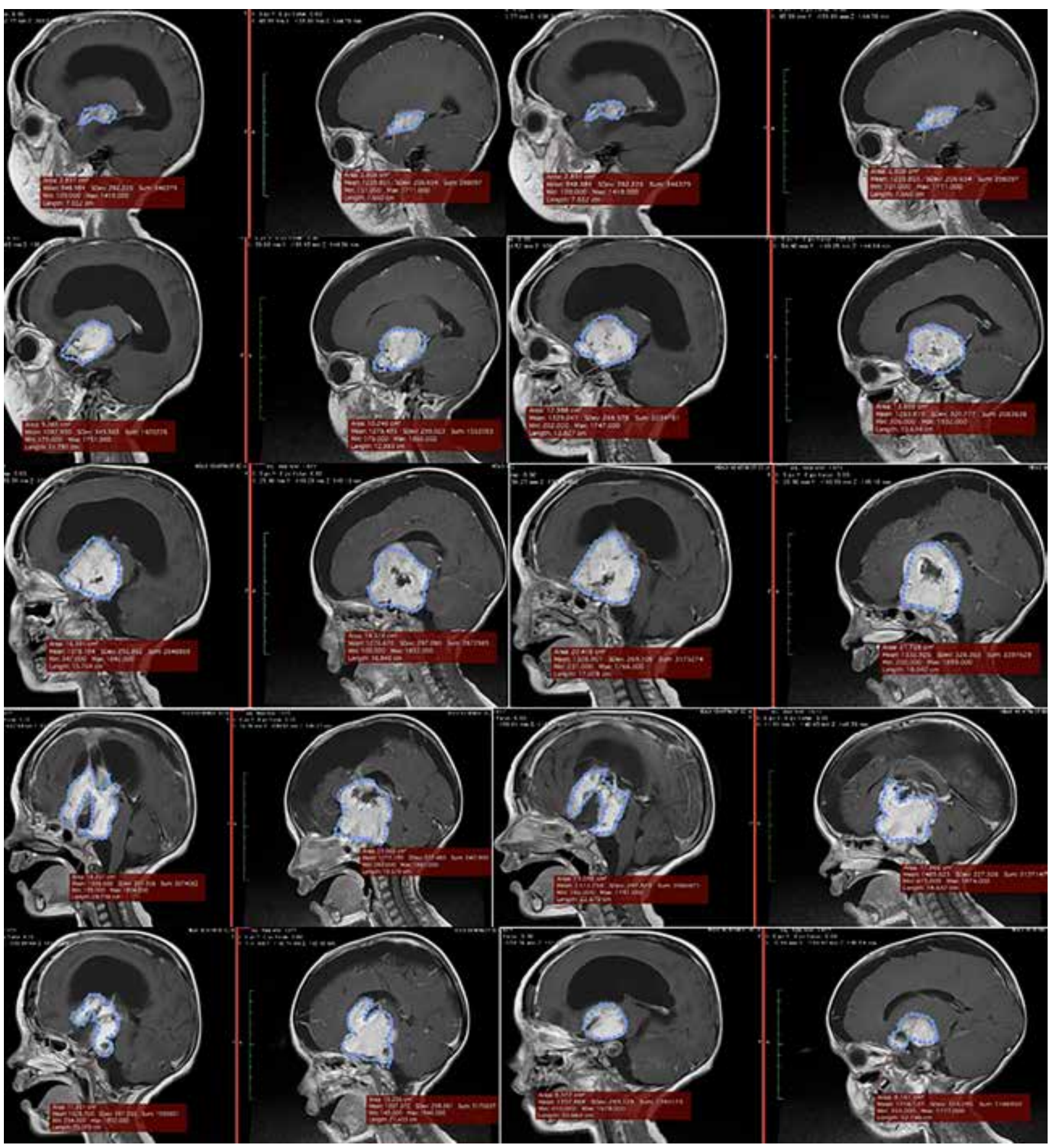

Fig. 4. T1-Constrast MRI of brain (sagittal) pre and post- second operation with volumetric calculation after partial removal of tumor)

although the diencephalic region and, notably, the hypothalamic/chiasmatic area are unquestionably the most frequently observed [6].

The clinical signs of PMA are similar to those of other juvenile brain tumors, such as developmental delay and altered level of awareness, in addition to hydrocephalus and widespread weakness, as well as localized neurological symptoms [6].

PMA and PA have distinct histologic characteristics. PMAs have a distinct myxoid matrix and monomorphous bipolar cells that typically radiate from arteries in an angiocentric manner. PMA contains very few eosinophilic granular bodies, Rosenthal fibers, plump «protoplasmic» cells, and a biphasic pattern, whereas PA has more. PMA features a monomorphous myxoid backdrop and angiocentric pattern, both of which are unusual in PA. Piloid cells are common in both tumor types. In contrast to normal PA, mitoses are frequent in PMA, and necrosis is not rare [1,7-9]. GFAP, S-100, and Vimentin immunopositivity is high in tumor cells and fibrillary background. Though the angiocentric/pseudorosette pattern is similar to ependymoma, the absence of EMA perinuclear dot expression in the current case of PMA distinguished it from ependymoma $[10,11]$.

$57 \%$ of malignancies were radiologically found in the hypothalamic/chiasmatic/third ventricular area. 


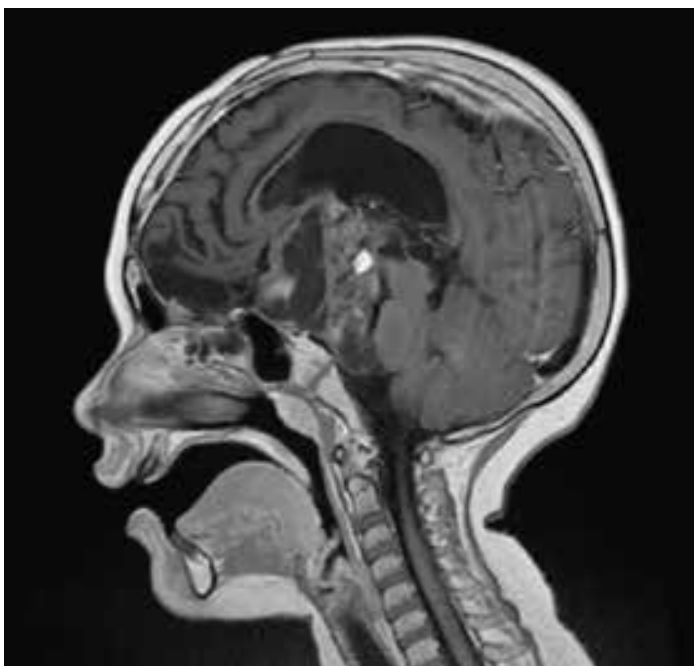

Fig. 5. Follow-up MRI after 3 years shows no progression of remaining lesion after partial removal and followed by chemotherapy and radiosurgery)

The remaining cancers were found in different parts of the brain, including the parietal lobe, temporal lobe, cerebellum, basal ganglia, and fourth ventricle. There was heterogeneous rim enhancement in $48 \%$ of the tumors, uniform enhancement in $43 \%$, and no enhancement in $9 \%$ of the tumors. In $24 \%$ of the cases, intratumoral bleeding was seen [3]. PMAs are most often seen in the hypothalamic/chiasmatic area, although they can also be found elsewhere in the neuraxis. However, unlike PA, they are generally solid and rarely contain cystic components. Some hypothalamic/chiasmatic PMAs are substantial, hefty masses that can reach into the temporal lobes. Almost all PMAs are nicely confined with minimal to no peritumoral edema. They are generally hypointense on T1-weighted images and hyperintense on T2-weighted images when scanned with MRI. They demonstrate either homogeneous or heterogeneous contrast enhancement. Intratumoral bleeding has been found in up to $25 \%$ of tumors in one study, which is greater than the percentage reported for PAs. In PMA, radiologic evidence of cerebral fluid dispersion is more prevalent than in PA $[8,12]$.

The treatment of PMA is still controversial and is heavily impacted by tumor site. The most significant predictor of a positive prognosis in children is gross total resection (GTR) of newly diagnosed tumors, assuming it can be performed without severe sequelae. GTR is achievable when the lesion is located in the cerebellar hemisphere and cerebral lobes distant from the eloquent area. However, if the lesion is located in the hypothalamic chiasmatic III ventricle area, the fourth ventricle, or another eloquent location, the excision rate is reduced [13]. CSF diversion by external drainage or VP shunt might be performed on a patient with hydrocephalus. In the case of persistent tumors, the role of adjuvant treatment is still debatable. Several studies have indicated chemotherapy as an adjuvant treatment for PMA. In situations of subtotal resection (STR) or partial resection (PR) and recurrence, adjuvant chemotherapy or radiation may be recommended. When non-enhancing solid tumors spread across the CSF space, they provide diagnostic issues. On a Fluid Attenuation Inversion Recovery (FLAIR) picture, non-enhancing CSF dispersion is more visible $[14,15]$. Chemotherapy is currently used sparingly for incurable or partly resected malignancies, and it may postpone the need for radiation in early infancy. Radiotherapy is often reserved for patients over the age of 5 who have had an initial surgical resection and have tumors near the midline [16].

This case report depicts a 2 year-6-month-old female patient with PMA who had partial resection of the tumor along with adjuvant dabrafenib treatment and radiosurgery, as the patient is old enough to receive radiation. A year following therapy, no progression of tumor was observed on MRI, and the patient is asymptomatic.

\section{Conclusion}

Pilomyxoid astrocytoma is a rare central nervous system tumor that has just been characterized and with few reports thus far, leading neurosurgeons and neurologists to a lack of knowledge of the tumor's behavior and, as a result, its therapy. Surgical intervention remains the first step, with complete resection being the goal. PMA has been reported as a more aggressive infancy tumor than PA, and its therapy is still debatable. Additional studies are necessary to better understand the clinical course of this tumor and, as a result, to enhance therapy options. The authors provide a case of a 2-year-and 6-month-old female patient with PMA who had partial resection with chemotherapy and radiosurgery and showed no indications of disease progression after a one-year follow-up.

\section{Recommendation}

When considering the diagnosis of PAs, histopathologists should search for characteristics of PMAs, since this discovery is significant for both the patient and the surgeon.

\section{Disclosure}

The authors declare no conflicts of interest with regard to the materials or techniques utilized in this investigation, as well as the conclusions stated in this work.

\section{Reference}

1. Gader G, Belkahla G, Karmani N, Saadaoui K, Rkhami M Kallel J, Zammel I, Badri M. Pediatric Cerebellar Pilomyxoid Astrocytoma: Clinical and Radiological Findings in Three Cases. Asian J Neurosurg. 2020 Apr 7;15(2):262-265. doi: 10.4103/ajns.AJNS_268_19

2. Karthigeyan M, Singhal $P$, Salunke $P$, Vasishta RK. Adult Pilomyxoid Astrocytoma with Hemorrhage in an Atypical Location. Asian J Neurosurg. 2019 Jan-Mar;14(1):300-303. doi: 10.4103/ajns.AJNS_164_18

3. Kim SH, Kang SS, Jung TY, Jung S. Juvenile pilomyxoid astrocytoma in the opticohypothalamus. J Korean Neurosurg Soc. 2010 Nov;48(5):445-7. doi: 10.3340/jkns.2010.48.5.445

4. Fomchenko EI, Reeves BC, Sullivan W, Marks AM, Huttner A, Kahle KT, Erson-Omay EZ. Dual activating FGFR1 mutations in pediatric pilomyxoid astrocytoma. Mol Genet Genomic Med. 2021 Feb;9(2):e1597. doi: 10.1002/mgg3.1597

5. Komotar RJ, Mocco J, Jones JE, Zacharia BE, Tihan T, Feldstein NA, Anderson RC. Pilomyxoid astrocytoma: diagnosis, prognosis, and management. Neurosurg Focus. 2005 Jun $15 ; 18(6 \mathrm{~A})$ :E7. 
6. Benyakhlef S, Tahri A, Khlifi A, Abdelouahab H, Imane K, Moufid $F$, Rouf $S$, Latrech $H$. Failure to Thrive Revealing a Pilomyxoid Astrocytoma: An Uncommon Case Report with Literature Review. Case Rep Pediatr. 2021 Sep 27;2021:6670585. doi: 10.1155/2021/6670585

7. Gupta K, Tewari MK, Salunke P. Pilocytic Astrocytoma with Gangliocytic Differentiation to Pilomyxoid Astrocytomaexpanding the Morphological Spectrum: Case Report and Literature Review. Asian J Neurosurg. 2018 OctDec;13(4):1193-1196. doi: 10.4103/ajns.AJNS_247_17

8. Ding C, Tihan T. Recent Progress in the Pathology and Genetics of Pilocytic and Pilomyxoid Astrocytomas. Balkan Med J. 2019 Jan 1;36(1):3-11. doi: 10.4274/balkanmedj.2018.1001

9. Arslanoglu A, Cirak B, Horska A, Okoh J, Tihan T, Aronson $L$, Avellino AM, Burger PC, Yousem DM. MR imaging characteristics of pilomyxoid astrocytomas. AJNR Am J Neuroradiol. 2003 Oct;24(9):1906-8

10. Kil JS, Lee KH, Eom KS, Kim TY. Intermediate Pilomyxoid Astrocytoma in the Cerebellum of a 5-Year-Old Boy. Brain Tumor Res Treat. 2018 Apr;6(1):39-42. doi: 10.14791/ btrt.2018.6.e6

11. Pruthi SK, Chakraborti S, Naik R, Ballal CK. Pilomyxoid astrocytoma with high proliferation index. J Pediatr Neurosci.
2013 Sep;8(3):243-6. doi: 10.4103/1817-1745.123694

12. Linscott LL, Osborn AG, Blaser S, Castillo M, Hewlett RH, Wieselthaler N, Chin SS, Krakenes J, Hedlund GL, Sutton CL. Pilomyxoid astrocytoma: expanding the imaging spectrum. AJNR Am J Neuroradiol. 2008 Nov;29(10):1861-6. doi: 10.3174/ajnr.A1233

13. Patibandla MR, Thotakura AK, Uppin M, Challa S, Addagada GC, Nukavarapu M. Parietal pilomyxoid astrocytoma with recurrence in 10 months: A case report and review of literature. Asian J Neurosurg. 2016 Jul-Sep;11(3):323. doi: 10.4103/1793-5482.145158

14. Pereira FO, Lombardi IA, Mello AY, Romero FR, Ducati LG, Gabarra RC, Zanini MA. Pilomyxoid astrocytoma of the brainstem. Rare Tumors. 2013 Apr 15;5(2):65-7. doi: 10.4081/ rt.2013.e17

15. Kim S, Kang M, Choi S, Kim DC. Pilomyxoid Astrocytoma Occurring in the Third Ventricle. J Clin Imaging Sci. 2015 Jul 31;5:41. doi: 10.4103/2156-7514.161853

16. Tjahjadi M, Arifin MZ, Sobana M, Avianti A, Caropeboka MS, Eka PA, Agustina H. Cystic pilomyxoid astrocytoma on suprasellar region in 7-year-old girl: Treatment and strategy. Asian J Neurosurg. 2015 Apr-Jun;10(2):154-7. doi: 10.4103/1793-5482.154989 